\title{
Pollutant dispersion in the urban environment
}

\author{
Matteo Carpentieri \\ Environmental Flow Research Centre (EnFlo), University of Surrey, GU2 7XH Guildford, UK \\ Tel. $+44(0) 1483686657$ \\ m.carpentieri@surrey.ac.uk
}

Published in: Reviews in Environmental Science and Bio-Technology (2013) - Vol. 12 (1) - pp. 5-8

DOI:10.1007/s11157-012-9305-8

\begin{abstract}
Flow and pollutant dispersion models are important elements for managing air quality in urban areas, to complement and, sometimes, even substitute monitoring. Developing fast and reliable parameterisations is necessary to improve the spatial and temporal resolutions of current mathematical prediction models. Recently there has been a growing interest in the so-called "neighbourhood scale" models, that offer relatively high spatial and temporal resolutions while keeping the needed computational resources at a minimum. This paper describes experimental and numerical simulations performed to explore the interaction of flow and pollutant dispersion with local building and street geometry. The methods developed may be useful as a way for cities to improve air quality management.
\end{abstract}

Keywords: Wind tunnel; CFD; Pollutant dispersion; Urban area; Neighbourhood scale.

\section{Introduction}

As many cities around the world become more congested, concerns increase over the level of urban air pollution generated and, in particular, its impact on local human health. In the majority of the developed world, legislation has already been introduced to the extent that local authorities are required by law to conduct regular Local Air Quality Reviews of key urban pollutants produced by industrial activity and/or road transport in order to introduce mitigation controls or to provide accurate public health warnings to those most vulnerable to the affect of poor air quality. Historically, monitoring has been used as a basis for the regulatory control of emission sources and as a tool to enforce air quality standards in urban and industrial areas. The use of modelling to support monitoring networks (or to replace them entirely in less polluted areas) has been introduced quite recently in legislation (e.g. EC Air Quality Framework Directive, 1996). Current state-of-the-art dispersion models have been developed for coarser scales and lower resolutions, and they are not capable of correctly reproducing the spatial and temporal distribution of air pollution within the urban environments where most people live. The neighbourhood scale (up to 1-2 km) allows for 
both a detailed reproduction of the phenomenon and the development of parameterised operational models (Britter and Hanna 2003). Neighbourhood scale models must take into account dispersion phenomena that occur in the urban canopy (defined as the region below roof level in dense urban areas). However, they cannot resolve the small-scale flow around each individual building, thus some kind of parameterisation must be attempted. Current approaches rely on empirical parameterisations derived from analytical studies and/or few experimental data gathered mostly on very simplified geometries (e.g. single 2D street canyon - i.e. idealized street flanked on both sides, symmetrically, by uninterrupted buildings of the same height), or full-scale measurements (usually very case specific).

Most of the urban canopy models make use of spatially averaged velocity profiles and, in some cases, even a single spatially averaged canopy velocity $\left(U_{C}\right.$, see Bentham and Britter 2003). These variables depend strongly on the local geometry. In order to describe the latter, parameters such as the mean building height $\left(H_{b}\right)$, the plan area index $\left(\lambda_{p}=A_{p} / A_{T}\right.$, where $A_{p}$ is the total building plan area and $A_{T}$ is the building lot area) and the frontal area index $\left(\lambda_{f}=\right.$ $A_{f} / A_{T}$, where $A_{f}$ is the total building frontal area) are generally used. However, several other geometric characteristics may have a strong influence, for example the building height variability. The distribution of pollutants in the canopy obviously strongly depends on the velocity field within the streets. Given the particular nature of the flow in dense urban areas, where recirculating flows are often observed in street canyons and in the wake of the buildings, pollutants tend to accumulate in the canopy, and thus the exchange mechanisms between the canopy and the flow above and between streets at intersections become very important (Caton et al. 2003; Carpentieri et al. 2012a). These fluxes are rarely measured in urban experiments (full scale or laboratory scale), especially the turbulent fluxes that are likely to be dominant for the vertical exchange processes.

\section{Working principle}

In order to bridge this research gap, both wind tunnel experiments and numerical simulations were carried out within the High Resolution Models for Flow and Pollutant Dispersion in Urban Areas (HRModUrb) project. The influence of the local geometry on the studied phenomena was studied by using two different geometrical models. They had the same "lambda parameters" ( $\lambda_{p}$ and $\left.\lambda_{f}\right)$ but different arrangements. The models are simple building arrays, but somewhat more realistic than the regular and staggered arrays of cubes generally used for physical modelling. In order to study the influence of height variability on flow and dispersion, both constant height and variable height models were employed. 

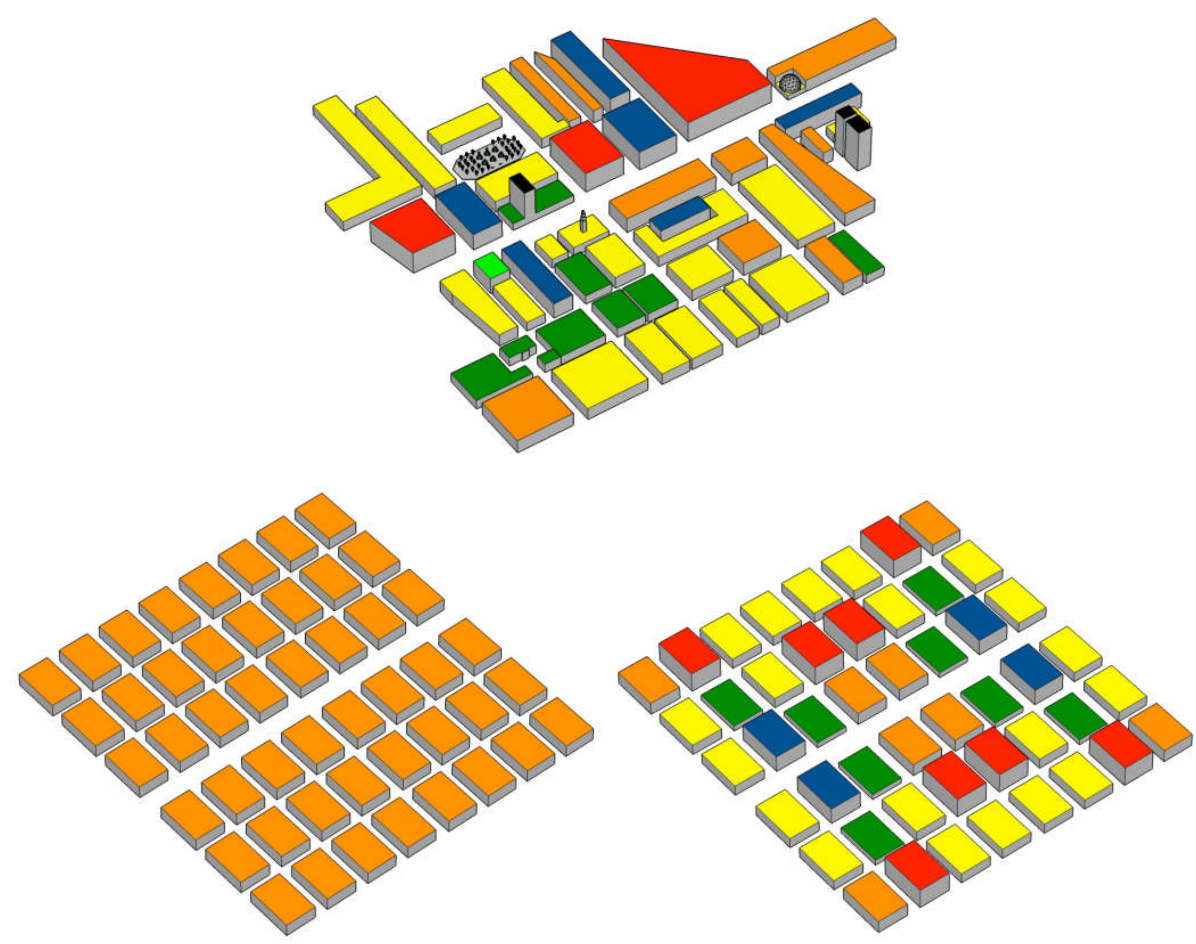

Fig 1. 3D rendering of the DAPPLE (top), SimpleC (bottom-left) and SimpleV (bottom-right) models.

The starting point for the design of the models was the 1:200 scale DAPPLE model (Arnold et al. 2004). The models designed for HRModUrb have two main intersecting streets (approximately matching those of the DAPPLE site in central London: Marylebone Road and Gloucester Place) and several smaller streets. The dimensions (width) of the main streets are, respectively: $220 \mathrm{~mm}$ and $110 \mathrm{~mm}$. The building blocks occupy an area of 230 x $350 \mathrm{~mm}^{2}$. In order to match the DAPPLE site $\lambda_{p}=0.54$, an array of $6 \times 8$ buildings was built, with the secondary streets width equal to $99 \mathrm{~mm}$ (Figure 1). As far as the vertical dimensions are concerned, two different models were employed in order to investigate the influence of the building height variability on the flow and dispersion phenomena. The simplest model (named 'SimpleC') had a constant building height $\left(H_{b}=102 \mathrm{~mm}\right.$, which is the mean building height of the central part of the DAPPLE model). Another model ('SimpleV') with variable building heights, matching the DAPPLE model standard deviation of building heights $\left(\sigma_{\mathrm{H}}=32 \mathrm{~mm}\right)$, was designed. Five different building heights were used; together with the building layout they were chosen to match the SimpleC mean building height and the DAPPLE model $\lambda_{\mathrm{f}}$ ( 0.24 for wind direction parallel to the $X$ axis, and 0.16 for wind direction parallel to the $Y$ axis).

In order to obtain the spatially averaged velocity profiles, the horizontal flow field was measured during two different sets of wind tunnel (WT) experiments using a 2-component 
laser Doppler anemometer (LDA, see Carpentieri et al. 2009, for a description of the technique). Tracer fluxes were measured by combining velocity and concentration measurements by means of a fast response flame ionisation detector (FFID, see Carpentieri and Robins, 2010). While the mean (advective) fluxes can be calculated by combining two sets of measurements (mean concentration and mean velocity) taken at different times, the turbulent fluxes require the simultaneous measurement of concentration and velocity. The measurement technique involves the coupled use of LDA and FFID (Carpentieri et al. 2012a). In order to integrate the wind tunnel experiments and to extend their scope, computational fluid dynamics (CFD) simulations were also carried out. Models based on the open-source library OpenFOAM (www.openfoam.org) were developed by using the widely accepted k- $\varepsilon$ turbulence model.

Results from the WT tests and CFD simulations highlighted the importance of differences in the geometry and the arrangement of the buildings, and this is reflected in the differences in the spatially averaged wind profiles calculated. As expected, a very high variability can be found within the canopy, while the velocity profiles tend to be more uniform above roof level (see Figure 2). These differences are not usually captured by current state-of-the art mathematical models. Similar conclusions can be drawn analyzing the tracer flux measurements.
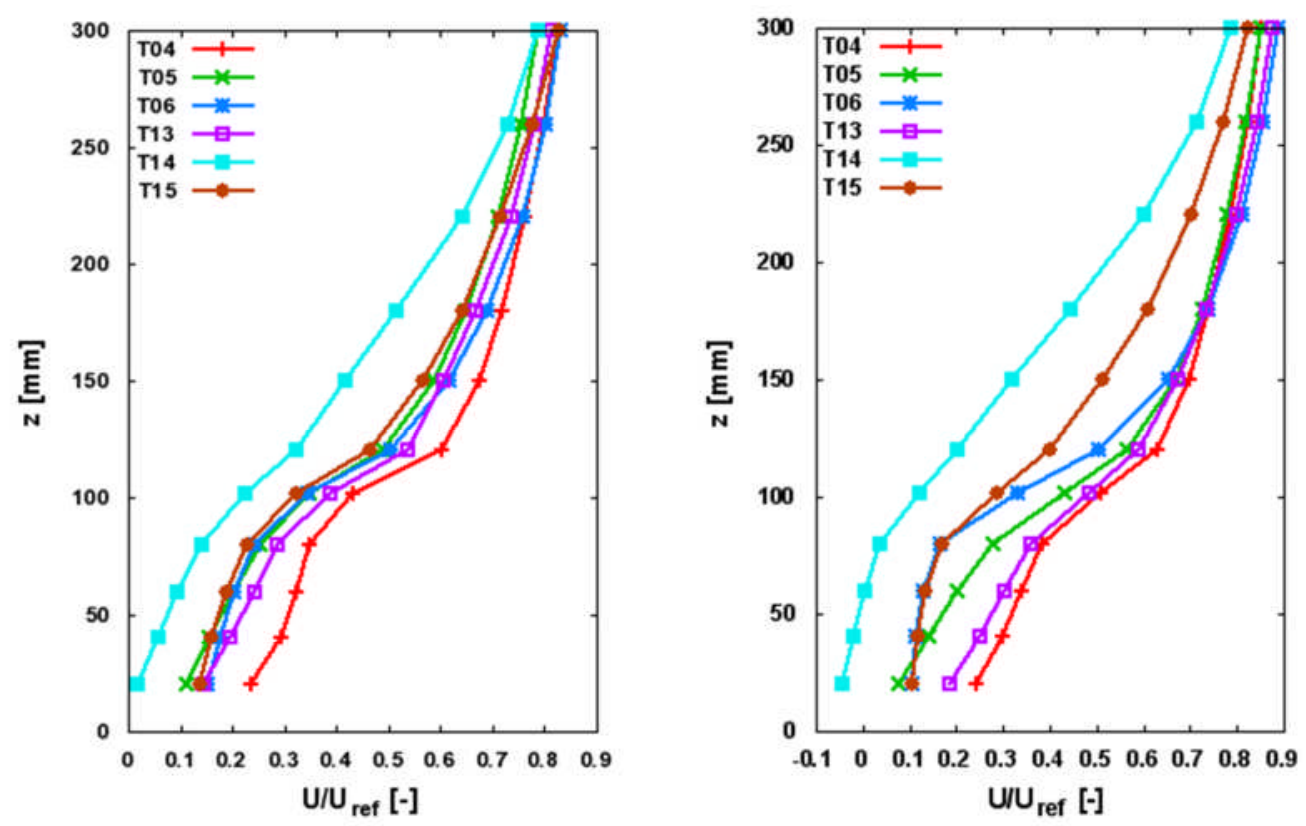

Fig 2. Spatially averaged velocity profiles for the six experimental cases (two models: SimpleC - T04, T05 and T06 - and SimpleV - T13, T14 and T15; and three wind directions: 0 - T04, T13 - 90 - T05, T14 - and 45 degrees - T06, T15). Wind tunnel results (left) and CFD simulations (right). 


\section{Applications}

The experimental and numerical data gathered during the project enable the development of new ways of parameterising urban morphology for operational flow and pollutant dispersion models. Linking flow and dispersion characteristics to the urban morphology has already been attempted in the past. The pioneer work by Grimmond and Oke (1999) in this field tried to relate some aerodynamic properties of urban areas (mainly, surface roughness and friction velocity) to a few geometrical characteristics (plane area density and frontal area density of the studied area and mean building height). Ratti et al. (2006) went even further by developing calculation techniques for deriving morphological properties from urban 3D databases (the three parameters mentioned above as well as other parameters, such as urban texture directionality and building height variability). However, they did not attempt to link those parameters to flow and dispersion characteristics, mainly because of the lack of experimental and numerical data. The experimental and numerical techniques applied during the HRModUrb project proved very promising and could be further developed do derive new parameterisations. The results database is particularly attractive to improve and validate the fast growing "street network modelling" approach, such as applied, for example, by the SIRANE model (Soulhac et al., 2011; Carpentieri et al., 2012b). Fast and reliable neighbourhood scale models could, in the future, enable new ways of managing air quality in cities, e.g. using real-time decision-making support tools based on integrated monitoring and modelling systems.

\section{Training of young researchers}

HRModUrb was an 'individual-driven' Marie Curie Intra-European Fellowship, supervised by Prof. Alan Robins. I was given the possibility of spending two years in one of the most renowned laboratories for studying environmental flows, the EnFlo lab at the University of Surrey. I had the chance of working with world-class facilities, such as the environmental boundary-layer wind tunnel, and learning new experimental techniques using advanced optical measurements methodologies. The project was also multi-disciplinary in nature, combining experimental fluid mechanics with numerical modelling, environmental physics and engineering. The experience and new skills gained during the Fellowship allowed me to further engage in the environmental fluids research as a postdoctoral research fellow. I am now working on developing interfaces between neighborhood scale parametric models and 
meteorological mesoscale models and on integrating modelling and monitoring for the development of real-time advanced air quality management systems.

\section{Acknowledgements}

The author acknowledges funding from the European Commission, FP7-People Programme (project HRModUrb) and help from the Marie Curie Fellows Association (mcfa.eu).

\section{References}

Arnold SJ, ApSimon H, Barlow J, Belcher S, Bell M, Boddy JW, Britter R, Cheng H, Clark R, Colvile RN, Dimitroulopoulou S, Dobre A, Greally B, Kaur S, Knights A, Lawton T, Makepeace A, Martin D, Neophytou M, Neville S, Nieuwenhuijsen M, Nickless G, Price C, Robins A, Shallcross D, Simmonds P, Smalley RJ, Tate J, Tomlin AS, Wang H, Walsh P (2004). Introduction to the DAPPLE air pollution project. Sci Total Environ 332:139-153.

Bentham T, Britter R (2003). Spatially averaged flow within obstacle arrays. Atmos Environ 37:2037-2043.

Britter R, Hanna S (2003). Flow and dispersion in urban areas. Ann Rev Fluid Mech 35:469- 496.

Caton F, Britter R, Dalziel S (2003). Dispersion mechanisms in a street canyon. Atmos Environ 37:393-702.

Carpentieri M, Hayden P, Robins A (2012a). Wind tunnel measurements of pollutant turbulent fluxes in urban intersections. Atmos Environ 46:669-674.

Carpentieri M, Robins A (2010). Tracer flux balance at an urban canyon intersection. Bound-Layer Meteorol $135: 229-242$.

Carpentieri M, Robins A, Baldi S (2009). Three-dimensional mapping of air flow at an urban canyon intersection. Bound-Layer Meteorol 133:277-296.

Carpentieri M, Salizzoni P, Robins A, Soulhac L (2012b). Evaluation of a neighbourhood scale, street network dispersion model through comparison with wind tunnel data. Environ Modell Softw 37:110-124.

EC Air Quality Framework Directive, 1996. European Commission, Council Directive 96/62/EC of 27 September 1996 on ambient air quality assessment and management. http://ec.europa.eu/environment/air/quality/legislation/existing_leg.htm.

Grimmond CSB, Oke TR (1999). Aerodynamic properties of urban areas derived from analysis of surface form. J Appl Meteorol 38:1262-1292.

Ratti C, Di Sabatino S, Britter RE (2006). Urban texture analysis with image processing techniques: winds and dispersion. Theor Appl Climatol 84:77-90.

Soulhac L, Salizzoni P, Perkins RJ (2011). The model SIRANE for atmospheric urban pollutant dispersion; Part I, presentation of the model. Atmos Environ 45:7379-7395. 